\title{
Renato Dullbecco: genes, cancer and epigenomics
}

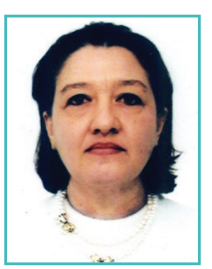

In 1986 Dr. Renato Dulbecco was one of the earliest proponents for sequencing of the human genome in order to guide human medical and disease research. He proposed that in the near future, the harmful effects of diseases could be treatable or even curable when the genes and the mutations that the genes harbored could be identified with certainty. This proposal commonly referred as the "human genome project" was based on concepts and ideas that he developed earlier.

In 1975 during the Nobel Prize acceptance awards ceremony, Dr. Dulbecco pioneered the idea of a cancer genome and transcriptome initiative proposing, "cancer is a disease of our genes" and suggesting that the cancer genome and cancer transcriptome would be generated by comparing the cancer cells with their corresponding healthy cells derived from the same patient. The nascent ideas generated from these sequencing proposals were the foundation of his two primary research focuses developed later. These were the identification of genes and pathways associated with normal cell differentiation compared to genes and pathways associated with abnormal cell regulation that resulted in cancer.

To understand cancer, he suggested one had to understand the normal differentiation process of stem cells, and in particular the genes that govern cell differentiation and tissue development. Stem cells have specific properties that are critical for cancer development and progression, such as significant potential for cellular proliferation and the property of chromatin remodeling, attributes required for cancer cells to adapt, evolve and modify themselves.

The characterization of normal stem cells and the origin of cancer stem cells were the focus of his research spanning almost two decades at the CNR-ITB. 